prevention, but The Health of the Nation charges us all with the duty to act. Collecting data in the form of case registers enables us to continue refining our understanding of risk factors for suicide and how these can change over time. We need to address suicide prevention for psychiatric patients, including the high risk period on discharge from hospital, and the role that malignant alienation may play (Watts \& Morgan, 1994): the Care Programme Approach may improve our response to these individuals. We must also devise means of reaching out to those who will die without having had contact with mental health services or their general practitioners. By auditing suicide in a systematic way clinical decision-making will be based on pooled experience not just on the cliniclan's memory of one or two cases. Comprehenstve registers of suicide provide clinictans with the opportunity of reviewing all cases in which they have had clinical involvement, so increasing the reliability of clinical experience. Overall, we need an attitude of vigilance about suicide risk, and of enthusiasm about pursuing initiatives based on research and clinical experience.

(Discussion of confidentiality and manpower will be found in part II and the references at the end of part II)

\title{
How to establish case registers: II. Non-fatal deliberate self-harm
}

\author{
J. Evans, C. Johnson, R. Stanton and H. G. Morgan
}

\begin{abstract}
The importance of cetcblithing registers for non-fotal dellberate selt-horm is cliscusesed along with their potentilat uses. Dellberate sell-harm (DSH) data for a single hoeplitel con bo dertved from the Accident and Emergency (ARE) casuctly cords, AaE on-line reglaters, mental hocith asecesements or general hoeplital in-patient detla. Tho relattve mertts of thees dillerent data sources are discusesed and locel methodis deecribed. Some data destred from the DSH regiter cre shown.
\end{abstract}

Patients who deliberately but non-fatally selfharm (DSH), utilise approximately 300 patientyears of acute care annually in England and Wales (Owens, 1990); in one study, 2.8\% had died by suicide within eight years of an attempt (Hawton \& Fagg, 1988). The costs to the individuals, their social networks, and the health service are enormous; and yet, in spite of extensive research, we are little further forward in identifying those at greatest risk, defining effective intervention strategles, or planning service provision based on research data. This paper describes a method of establishing a hospital-based reglster of DSH for use in audit and research.
Table 1. Sources of data for DSH register

\begin{tabular}{|c|}
\hline 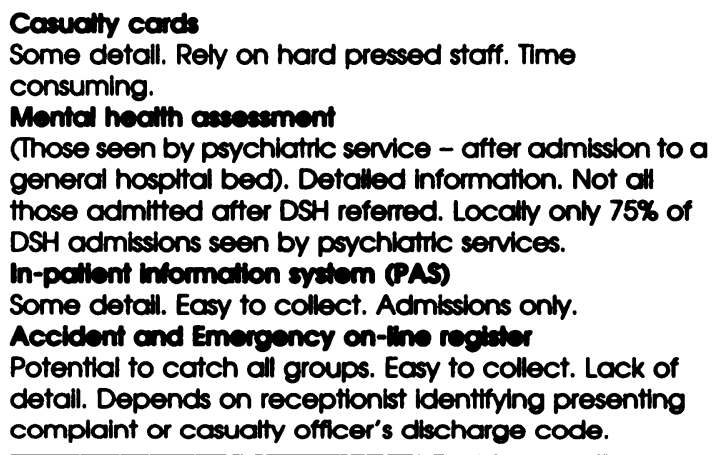 \\
\hline
\end{tabular}

\section{Data sources (Table I)}

Mental health assessments of those admitted following deliberate self-harm

A local audit indicated that $75 \%$ of those admitted following an episode of DSH are seen for a mental health assessment within 24 hours of admission. This provides an easily collected record, with 
detailed information, but one that is not complete.

\section{General hospital in-patient admission data}

Hospitals now employ coders to study patient records and record codes after discharge on the hospital information system. An enquiry can be made with most information systems to select for the ICD codes relevant to DSH (self inflicted injury (ICD-9; E950-E959) and injury undetermined whether accidentally or purposely inflicted (ICD-9; E980-989)) and this information downloaded onto a floppy disc to complle the case register. Locally these data become available six weeks after discharge, following organisation of the hospital data for the South West using the Centrelink System at regional headquarters. This includes all those admitted who have selfharmed, but will provide less information than the mental health assessments. These data include a higher proportion of patients who have taken overdoses, as those who are discharged or self-discharge without overnight admission from accident and emergency (A\&E) departments, many of whom have self-lacerated, are excluded.

\section{Accident and Emergency departments}

The advantage of basing the register on $A \& E$ department data is that those who are discharged (e.g. self-laceration), and those who self-discharge are included. It has been estimated that 20-30\% of patients who present with DSH are discharged although there is considerable regional variation in this proportion.

Data can be collected in several ways. In an A\&E department without computerised records that is not too busy, an alphabetical card system can be obtained by photocopying the casualty cards of those identified by casualty staff as presenting following an episode of DSH.

Alternatively, where departments have computerised on-line registers, an enquiry can search for identifiers in the 'presenting complaint', or for casualty officers' diagnostic codes if these are used.
The main limitation of this system is the amount of information that can be collected. which is less than that for a card system and may exclude important variables (for example. method of self-harm). However, once established it requires few resources to maintain.

In Bristol we have based the DSH register (Table 2) on the A\&E department on-line register. cross-checked and expanded with general hospital in-patient data from the hospital information system. It has been found that a high percentage $(>95 \%)$ of the diagnostic information collected in routine hospital statistics is correctly recorded (Seller et al, 1990) so we feel confident in using this method of data collection.

To select those who have presented following an episode of DSH it is necessary to ask the A\&E receptionists to standardise their terminology for the 'presenting complaint'. For our register, the code 'S/I' for 'Self-inflicted' was chosen as it was both meaningful to the staff and distinctive.

We then approached the information technology department for assistance in writing a programme to search for ' $S / \mathrm{I}$ ' in the presenting complaint. To ensure that cases were not being missed we included variations of the term 'overdose' in the search, as well as the casualty officer's discharge code for DSH (code 11). Weekly information is downloaded onto a computerised spreadsheet. Some editing of data is required to refine the register. By using a spread sheet in which data can easily be sorted it is possible to check for obvious inaccuracies, removing duplications or irrelevant episodes (such as attending a dressing clinic). Sorting by surname will provide an alphabetical register and enable identification of multiple attendances by a single individual.

General hospital in-patient admission data are available monthly, also on a computerised spreadsheet. Combining this with the register compiled from A\&E data and removing those who appear twice produces the final register. Uses of the register can be decided locally. Providing information for suicide audit has been discussed, and comparing rates across the locality by using addresses, identifying multiple repeaters, calculating admission rates from casualty to general hospital in-patient beds and

Table 2. Bristol Royal Infirmary DSH register (1993)

\begin{tabular}{lr}
\hline Total number of eplsodes & 991 \\
Total number of individuals & 642 \\
Total number of eplsodes resulting in discharge from A\&E & 279 \\
Total number of eplsodes leading to hospltal admission & 712 \\
Of those admitted, number of eplsodes involving an overdose & 627 \\
Of those admitted, number of eplsodes involving paracetamol overdose & 274 \\
Of those admilted, number of eplsodes involving other methods of DSH & 85 \\
Number of indlividuals who appeared more than once on the register & 102 \\
\hline
\end{tabular}

"N.B. This is not the repettition rate. 
repetition rates are all important in audit and planning of local policy.

\section{Confidentiality: registers of suicide and DSF}

Confidentiality must be respected. For computerised registers it is necessary to discuss issues of confidentiality and legality with the local Data Protection Officer. Registration under the Data Protection Act (1984), for research and registers is necessary, and Trusts or Health Authorities should already be registered to collect this information.

Protocols should be submitted to local $\mathbf{R e}$ search Ethics Committees. In our case, consultant approval and consent through the local medical committee were considered sufficient; any further research project using the registers would require separate ethical committee approval.

\section{Manpower: registers of suicide and DSF}

To set up both registers in Bristol required input by a psychiatrist for two sessions a week for a month to negotiate with $A \& E$ consultant and reception staff, the hospital information technology department and coroners. Once established both registers can be maintained by a secretary spending one session a week editing the register and informing those responsible for organising audit and one session a month by an audit coordinator to examine the coroner's files. This will vary depending on local circumstances and the number of sudden and unexpected deaths and the number of hospital attendances for DSH.

\section{Comment}

DSH is associated with considerable morbidity and heavy use of services, yet it is not always recognised as a pressing public health problem. A DSH case register highlights the urgent need to address aspects of the problem, in particular, the fate of those who self-discharge or are discharged from A\&E departments, and those who frequently repeat. The recent identification of the increased risk of suicide among the young substance abusers admitted after DSH illustrates the value of collecting these data (Hawton et al, 1993). Many individuals are or have been in contact with a wide range of professionals but coordination of care and communication are often lacking. Although the DSH episode may be impulstve, it commonly arises in the context of mounting social difficulties, and so we need to find ways of intervening at an early stage.

\section{References}

DEPARTMENT OF HEALTH (1992) The Health of the Nation: A Strategy for Health in England. London: HMSO.

HAWTON, K. \& FAGG, J. (1988) Suicide and other causes of death following attempted suicide. Brttish Journal of Psychiatry, 182, 359-366.

- . - . PLATT, S., et al (1993) Factors assoclated with sulcide after parasuicide in young people. Brttish Medical Journal, S08, 1641-1644.

MoRGAN, H. G. (1994) Clinical Audit of Sulcide and Other Unexpected Deaths. NHS Management Executtve.

Office of POpUlation Censuses and SuRvers (1991) Mortality Statistics for England and Wales. 1980 and 1991 (DH2, No. 17). London: HMSO.

OWENS, D. (1990) Self-harm patients not admitted to hospital. Journal of the Royal College of Physictans, 24. 281-283.

SELLER, C., GOLDACRE, M. J. \& HAWTON, K. (1990) Reliability of routine hospital data on poisoning as measures of deliberate self poisoning in adolescents. Journal of Epidemiology and Communtty Health, 44, 313-315.

WATTS, D. \& MORGAN, H. G. (1994) Malignant alienation. British Journal of Psychlatry, 164, 11-15.

Wortd Health Organization (1978) Mental Disorders: Glossary and Guide to thetr Classification in Accordance with the Ninth Revision of the International Classification of Diseases (ICD-9). Geneva: WHO.

*J. Evans, Lecturer, C. Johnson, Lecturer, R. Stanton, Research Fellow; and H. G. Morgan, Professor, Department of Mental Health, University of Bristol, 41 St Michael's Hill, Bristol BS2 8DZ

*Correspondence 